\title{
Food \\ Technology and Biotechnology
}

\author{
Vol. 42 \\ Contents, Author and Subject Index
}

\author{
ZAGREB \\ CROATIA \\ 2004
}




\section{CONTENTS}

\section{ORIGINAL SCIENTIFIC PAPERS}

Simón J. Téllez-Luis, Juan J. González-Cabriales, José A. Ramírez and Manuel Vázquez Production of Transglutaminase by Streptoverticillium ladakanum NRRL-3191 Grown on Media Made from Hydrolysates of Sorghum Straw . . . . . . . . . . . . . . . . . . (1)

Fabiana Ribeiro Vianna, Carolina Schaper Bizzotto, Disney Ribeiro Dias, Afonso L. Oliveira and Marialice Pinto Coelho Silvestre

Bovine Blood Constituents as Fat Replacers in Ham Pâté. . . . . . . . . . . . . . . . . . (1)

Iztok Klenar, Marin Berovič and Mojmir Wondra

Phenolic Compounds from the Fermentation of Cultivars Cabernet Sauvignon and Merlot

from the Slovenian Coastal Region . . . . . . . . . . . . . . . . . . . . . . (1)

Simón J. Téllez-Luis, José A. Ramírez and Manuel Vázquez

Production of Transglutaminase by Streptoverticillium ladakanum NRRL-3191 Using Glycerol

as Carbon Source . . . . . . . . . . . . . . . . . . . . . . . . . . . . . . . . . (2)

Mohammed S. El-Katatny, Hesham M. El-Komy, Gehan M. Shaban, Ahmed M. A. Hetta and

Momein H. El-Katatny

Effect of Benomyl on Chitinase and $\beta-1,3-$ Glucanase Production by Free and Alginate

Encapsulated Trichoderma harzianum . . . . . . . . . . . . . . . . . . . . (2)

Clemencia Chaves López, Emanuele Boselli, Andrea Piva, Maurice Ndaghijimana,

Antonello Paparella, Giovanna Suzzi and Dino Mastrocola Influence of Quinoxyfen Residues on Saccharomyces cerevisiae Fermentation of Grape Musts. . . . .

Tvrtko Ahel, Ivan Mijatović, Marin Matošić and Marijan Ahel

Nanofiltration of a Landfill Leachate Containing Pharmaceutical Intermediates from Vitamin C

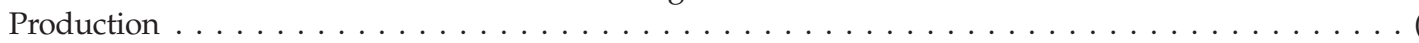

Marcin Skowronek and Jan Fiedurek

Optimisation of Inulinase Production by Aspergillus niger Using Simplex and Classical Method . . (3)

Basa Janakiram Naveena, Mohammad Altaf, Kalva Bhadrayya and Gopal Reddy

Production of $\mathrm{L}(+)$ Lactic Acid by Lactobacillus amylophilus GV6 in Semi-Solid State

Fermentation Using Wheat Bran . . . . . . . . . . . .

Mario Rodríguez-Monroy, José Luis Trejo-Espino, Antonio Jiménez-Aparicio,

Ma. de la Luz Morante, Ma. Luisa Villarreal and Gabriela Trejo-Tapia

Evaluation of Morphological Properties of Solanum chrysotrichum Cell Cultures in a Shake

Flask and Fermentor and Rheological Properties of Broths. . . . . . . . . . . . . . . (3)

Gintare Gulbiniene, Laima Kondratiene, Tautvile Jokantaite, Elena Serviene, Vytautas Melvydas and Giedre Petkuniene

Occurrence of Killer Yeast Strains in Fruit and Berry Wine Yeast Populations.

Margherita Paraggio

Biodiversity of a Natural Population of Saccharomyces cerevisiae and Hanseniaspora uvarum from

Aglianico del Vulture. . . . . . . . . . . . . . . . .

Ayumi Abe, I-Nengah Sujaya, Teruo Sone, Kozo Asano and Yuji Oda

Microflora and Selected Metabolites of Potato Pulp Fermented with an Indonesian Starter

Ragi Tapé . . . . . . . . . . . . . . . . . .

Mojca Narat, Ajda Biček, Robert Vadnjal and Dušan Benčina

Production, Characterization and Use of Monoclonal Antibodies Recognizing IgY Epitopes

Shared by Chicken, Turkey, Pheasant, Peafowl and Sparrow. . . . . . . . . . . . . . . . . (3)

Snežana Stanković, Slobodan Jović and Jelena Živković

Bentonite and Gelatine Impact on the Young Red Wine Coloured Matter . . . . . . . . . . . . . (3)

Arantza Gómez de Segura, Miguel Alcalde, Nieves López-Cortés, Francisco J. Plou and Antonio Ballesteros

Modulating the Synthesis of Dextran with the Acceptor Reaction Using Native 
Andrés R. Alcántara, Pablo Domínguez de María, María Fernández, María José Hernaíz, José María Sánchez-Montero and José Vincente Sinisterra Resolution of Racemic Acids, Esters and Amines by Candida rugosa Lipase in Slightly Hydrated Organic Media . . . . . . . . . . . . . . . . . . . . . . . . . . . . . . . . . . (4)

Regina A. C. Gonçalves, André L. M. Porto, Lucimar Pinheiro, José R. Cagnon,

Gilson P. Manfio and Anita J. Marsaioli Multibioreaction Methodology for Baeyer-Villiger Monooxygenase Monitoring .

\section{PRELIMINARY COMMUNICATIONS}

Emine Alben and Osman Erkmen

Production of Citric Acid from a New Substrate, Undersized Semolina, by Aspergillus niger. . . . . (1)

Leonard Vuyani Mabinya, John Morgan Brand, Ndjoko Yei Osée Muyima and

Ganka Lubenova Pironcheva

Kinetic Properties of $\alpha$-Galactosidase and the Localization of Total Proteins in Erwinia

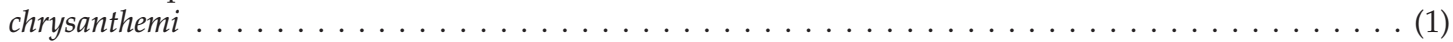

Sibel Fadiloğlu, Osman Erkmen and Gülten S,keroğlu

Thermal and Carbon Dioxide Inactivation of Alkaline Phosphatase in Buffer and Milk . . . . . . (1)

Hrvoje Lepeduš, Vera Cesar and Marijana Krsnik-Rasol

Guaiacol Peroxidases in Carrot (Daucus carota L.) Root . . . . . . . . . . . . . . . . . . . . . . . (1)

Andrea Caridi, Antonio Cufari, Raffaele Lovino, Rosanna Palumbo and Idolo Tedesco

Influence of Yeast on Polyphenol Composition of Wine . . . . . . . . . . . . . . . . (1)

Fabiano Cleber Bertoldi, Ernani S. Sant'Anna and Luiz H. Beirão

Reducing the Bitterness of Tuna (Euthynnus pelamis) Dark Meat with Lactobacillus casei subsp.

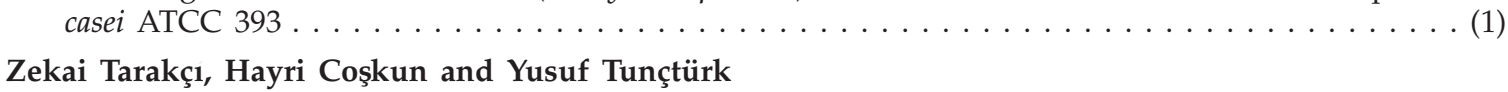

Some Properties of Fresh and Ripened Herby Cheese, a Traditional Variety Produced in Turkey . (1)

Dimitris P. Makris and Panagiotis Kefalas

Carob Pods (Ceratonia siliqua L.) as a Source of Polyphenolic Antioxidants. . . . . . . . . . . . . . . (2)

Marica Rakin, Josip Baras and Maja Vukasinovic

The Influence of Brewer's Yeast Autolysate and Lactic Acid Bacteria on the Production of a

Functional Food Additive Based on Beetroot Juice Fermentation. . . . . . . . . . . . . . . (2)

Vesna Stehlik-Tomas, Vlatka Gulan Zetić, Damir Stanzer, Slobodan Grba and Nada Vahčić Zinc, Copper and Manganese Enrichment in Yeast Saccharomyces cerevisiae . . . . . . . . . . . . . . (2)

Višnja Šverko, Sandra Sobočanec, Tihomir Balog, Miroslav Colić and Tatjana Marotti Natural Micronised Clinoptilolite and Clinoptilolite Mixtures with Urtica dioica L. Extract as Possible Antioxidants. . . . . . . . . . . . . . . . . . . . . .

Paolo Torre, Danilo De Faveri, Patrizia Perego, Attilio Converti, Paolo Barghini,

Maurizio Ruzzi and Fabrícia P. Faria Selection of Co-Substrate and Aeration Conditions for Vanillin Production

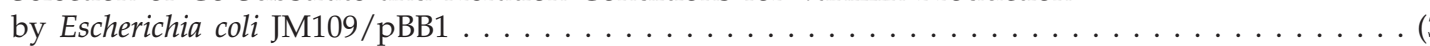

\section{SCIENTIFIC NOTES}

Carla J. S. M. Silva, Fernanda Sousa, Georg Gübitz and Artur Cavaco-Paulo

Chemical Modifications on Proteins Using Glutaraldehyde.

Nada Kolb, Luciana Vallorani, Nada Milanović and Vilberto Stocchi Evaluation of Marine Algae Wakame (Undaria pinnatifida) and Kombu (Laminaria digitata japonica) as Food Supplements . . . . . . . . . . . . . . . . . . . (1)

Osman Erkmen and Hüseyin Bozkurt Quality Characteristics of Retailed Sucuk (Turkish Dry-Fermented Sausage) . . . . . . . . . . . . (1)

sil Seyis and Nilufer Aksoz

Production of Lactase by Trichoderma sp.

Nuno Ratola, Joaquim Luís Faria and Arminda Alves Analysis and Quantification of trans-Resveratrol in Wines from Alentejo Region (Portugal) ..... . 
Yueming Jiang, Xinguo Su, Xuewu Duan, Wenbin Lin and Yuebiao Li

Anoxia Treatment for Delaying Skin Browning, Inhibiting Disease Development and

Maintaining the Quality of Litchi Fruit $\ldots \ldots \ldots \ldots \ldots \ldots \ldots \ldots \ldots \ldots$ (2)

Huaqiang Dong, Yueming Jiang, Yuehua Wang, Ruang Liu and Haoning Guan

Effects of Hot Water Immersion on Storage Quality of Fresh Broccoli Heads . . . . . . . . . . . . (2)

\section{REVIEWS}

Milan Sak-Bosnar, Zorana Grabarić and Božidar S. Grabarić

Surfactant Sensors in Biotechnology Part 1 - Electrochemical Sensors . . . . . . . . . . . . . . . (3)

Milan Sak-Bosnar, Zorana Grabarić and Božidar S. Grabarić

Surfactant Sensors in Biotechnology Part 2 - Non-Electrochemical Sensors. . . . . . . . . . . . . . . (3)

Srđan Novak, Vesna Zechner-Krpan and Vladimir Marić

Regulation of Maltose Transport and Metabolism in Saccharomyces cerevisiae. . . . . . . . . . . . . (3)

Joseph Gomes and Walter Steiner

The Biocatalytic Potential of Extremophiles and Extremozymes. . . . . . . . . . . . . . . (4)

Zhibin Liu, Roland Weis and Anton Glieder

Enzymes from Higher Eukaryotes for Industrial Biocatalysis . . . . . . . . . . . . . . . (4)

Marek Adamczak and Sajja Hari Krishna

Strategies for Improving Enzymes for Efficient Biocatalysis . . . . . . . . . . . . . . . . (4)

Jean-Louis Reymond

Spectrophotometric Enzyme Assays for High-Throughput Screening . . . . . . . . . . . . . . . . (4)

Sebastián Torres and Guillermo R. Castro

Non-Aqueous Biocatalysis in Homogeneous Solvent Systems .

Nadia Krieger, Tej Bhatnagar, Jacques C. Baratti, Alessandra M. Baron, Valéria M. de Lima and David Mitchell

Non-Aqueous Biocatalysis in Heterogeneous Solvent Systems. . . . . . . . . . . . . . . . . . (4)

Martin H. Fechter and Herfried Griengl

Hydroxynitrile Lyases: Biological Sources and Application as Biocatalysts . . . . . . . . . . . . . (4)

J. Augusto R. Rodrigues, Paulo J. S. Moran, Gelson J. A. Conceição and Lucídio C. Fardelone Recent Advances in the Bioacatalytic Asymmetric Reduction of Acetophenones and $\alpha, \beta$-Unsaturated Carbonyl Compounds

Ramesh N. Patel

Biocatalytic Synthesis of Chiral Pharmaceutical Intermediates

Mario Aguedo, Mai Huong Ly, Isabel Belo, José A. Teixeira, Jean-Marc Belin and Yves Waché

The Use of Enzymes and Microorganisms for the Production of Aroma Compounds from Lipids (4)

\section{APPENDIX}

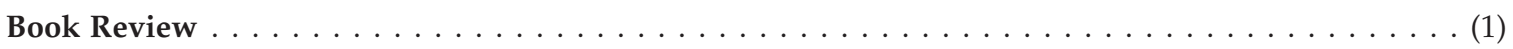

In memoriam

Prof. Dr. Sc. Branimir Zamola (1935-2003) .

Nadia Krieger, Walter Steiner and David Mitchell

Foreword: Frontiers in Biocatalysis . . . . . . . . . . . . . . . . . . (4) 


\section{AUTHOR INDEX}

Abe, Ayumi

(3) 169

Adamczak, Marek . . . . . . . . . . . (4) 251

Aguedo, Mario . (4) 327

Ahel, Marijan . . . . . . . . . . . . . (2) 99

Ahel, Tvrtko. . . . . . . . . . . . . . . . (2) 99

Aksoz, Nilufer . . . . . . . . . . . . . (2) 121

Alben, Emine . . . . . . . . . . . . . (1) 19

Alcalde, Miguel . . . . . . . . . . . . (4) 337

Alcántara, Andrés R. . . . . . . . . . . (4) 343

Altaf, Mohammad . . . . . . . . . . . (3) 147

Alves, Arminda . . . . . . . . . . . . (2) 125

Asano, Kozo. . . . . . . . . . . . . . (3) 169

Ballesteros, Antonio . . . . . . . . . . (4) 337

Balog, Tihomir. . . . . . . . . . . . . (3) 189

Baras, Josip . . . . . . . . . . . . . . . (2) 109

Baratti, Jacques C. . . . . . . . . . . . . (4) 279

Barghini, Paolo . . . . . . . . . . . . (3) 193

Baron, Alessandra M. . . . . . . . . . . . (4) 279

Beirão, Luiz H. . . . . . . . . . . . . . (1) 41

Belin, Jean-Marc . . . . . . . . . . . . (4) 327

Belo, Isabel . . . . . . . . . . . . . . (4) 327

Benčina, Dušan . . . . . . . . . . . . (3) 175

Berovič, Marin . . . . . . . . . . . . . (1) 11

Bhadrayya, Kalva . . . . . . . . . . . (3) 147

Bhatnagar, Tej . . . . . . . . . . . . . (4) 279

Biček, Ajda . . . . . . . . . . . . . . (3) 175

Boselli, Emanuele . . . . . . . . . . . (2) 89

Bozkurt, Hüseyin . . . . . . . . . . . (1) 63

Brand, John Morgan . . . . . . . . . (1) 23

Cagnon, José R. . . . . . . . . . . . . . . . . . (4) 355

Caridi, Andrea. . . . . . . . . . . . . (1) 37

Castro, Guillermo R. . . . . . . . . . . (4) 271

Cavaco-Paulo, Artur . . . . . . . . . . (1) 51

Cesar, Vera . . . . . . . . . . . . . (1) 33

Chaves López, Clemencia . . . . . . . . (2) 89

Cleber Bertoldi, Fabiano. . . . . . . . . (1) 41

Colić, Miroslav. . . . . . . . . . . . . (3) 189

Conceição, Gelson J. A. . . . . . . . . . (4) 295

Converti, Attilio . . . . . . . . . . . . (3) 193

Coşkun, Hayri . . . . . . . . . . . . . (1) 47

Cufari, Antonio . . . . . . . . . . . . (1) 37

Domínguez de María, Pablo . . . . . . . (4) 343

Dong, Huaqiang . . . . . . . . . . . . (2) 135

Duan, Xuewu . . . . . . . . . . . . . . (2) 131

El-Katatny, Mohammed S. . . . . . . . . (2) 83

El-Katatny, Momein H. . . . . . . . . . (2) 83

El-Komy, Hesham M. . . . . . . . . . . (2) 83

Erkmen, Osman . . . . . . . . . . . . (1) $19,27,63$

Fadiloğlu, Sibel . . . . . . . . . . . . (1) 27

Fardelone, Lucídio C. . . . . . . . . . . . . . . (4) 295

Faria, Joaquim Luís. . . . . . . . . . . (2) 125

Faria, Fabrícia P. . . . . . . . . . . . . (3) 193

De Faveri, Danilo . . . . . . . . . . . (3) 193
Fechter, Martin H. . . . . . . . . . . . . . . (4) 287

Fernández, María . . . . . . . . . . . (4) 343

Fiedurek, Jan . . . . . . . . . . . . . . . . . . (3) 141

Glieder, Anton. . . . . . . . . . . . . (4) 237

Gomes, Joseph. . . . . . . . . . . . . . (4) 223

Gómez de Segura, Arantza . . . . . . . (4) 337

Gonçalves, Regina A. C. . . . . . . . . (4) 355

González-Cabriales, Juan J. . . . . . . . . (1) 1

Grabarić, Božidar S. . . . . . . . . . . (3) 197,207

Grabarić, Zorana . . . . . . . . . . . . . . . (3) 197,207

Grba, Slobodan . . . . . . . . . . . . (2) 115

Griengl, Herfried. . . . . . . . . . . . (4) 287

Guan, Haoning . . . . . . . . . . . . . . (2) 135

Gübitz, Georg . . . . . . . . . . . . . (1) 51

Gulan Zetić, Vlatka. . . . . . . . . . . (2) 115

Gulbiniene, Gintare. . . . . . . . . . . (3) 159

Hernaíz, María José ․ . . . . . . . . . (4) 343

Hetta, Ahmed M. A. . . . . . . . . . . (2) 83

Jiang, Yueming. . . . . . . . . . . . . (2) 131,135

Jiménez-Aparicio, Antonio. . . . . . . . (3) 153

Jokantaite, Tautvile . . . . . . . . . . . . (3) 159

Jović, Slobodan . . . . . . . . . . . . . . (3) 183

Kefalas, Panagiotis . . . . . . . . . . . (2) 105

Klenar, Iztok. . . . . . . . . . . . . . (1) 11

Kolb, Nada . . . . . . . . . . . . . . (1) 57

Kondratiene, Laima. . . . . . . . . . . (3) 159

Krieger, Nadia . . . . . . . . . . . . . . . (4) 219,279

Krishna, Sajja Hari . . . . . . . . . . . (4) 251

Krsnik-Rasol, Marijana . . . . . . . . . (1) 33

Lepeduš, Hrvoje . . . . . . . . . . . . (1) 33

Li, Yuebiao . . . . . . . . . . . . . . . . . . (2) 131

de Lima, Valéria M. . . . . . . . . . . (4) 279

Lin, Wenbin . . . . . . . . . . . . . . (2) 131

Liu, Ruang . . . . . . . . . . . . . . (2) 135

Liu, Zhibin . . . . . . . . . . . . . . (4) 237

López-Cortés, Nieves . . . . . . . . . . (4) 337

Lovino, Raffaele . . . . . . . . . . . . (1) 37

Lubenova Pironcheva, Ganka . . . . . . (1) 23

Ly, Mai Huong . . . . . . . . . . . . (4) 327

Makris, Dimitris P. . . . . . . . . . . . (2) 105

Manfio, Gilson P.. . . . . . . . . . . . (4) 355

Marić, Vladimir . . . . . . . . . . . . . . (3) 213

Marotti, Tatjana . . . . . . . . . . . . . . . (3) 189

Marsaioli, Anita J. . . . . . . . . . . . (4) 355

Mastrocola, Dino. . . . . . . . . . . . . . (2) 89

Matošić, Marin. . . . . . . . . . . . . (2) 99

Melvydas, Vytautas. . . . . . . . . . . (3) 159

Mijatović, Ivan. . . . . . . . . . . . . (2) 99

Milanović, Nada . . . . . . . . . . . . (1) 57

Mitchell, David . . . . . . . . . . . . (4) 219,279

Moran, Paulo J. S. . . . . . . . . . . . . . . (4) 295 
Morante, Ma. de la Luz. . . . . . . . . (3) 153

Muyima, Ndjoko Yei Osée. . . . . . . . (1) 23

Narat, Mojca . . . . . . . . . . . . . . . . . . (3) 175

Naveena, Basa Janakiram . . . . . . . . (3) 147

Ndaghijimana, Maurice . . . . . . . . . . . (2) 89

Novak, Srđan . . . . . . . . . . . . . (3) 213

Oda, Yuji . . . . . . . . . . . . . . . (3) 169

Oliveira, Afonso L. . . . . . . . . . . . . (1) 5

Palumbo, Rosanna . . . . . . . . . . . (1) 37

Paparella, Antonello . . . . . . . . . . (2) 89

Paraggio, Margherita . . . . . . . . . . (3) 165

Patel, Ramesh N. . . . . . . . . . . . (4) 305

Perego, Patrizia . . . . . . . . . . . . (3) 193

Petkuniene, Giedre . . . . . . . . . . . . (3) 159

Pinheiro, Lucimar . . . . . . . . . . . (4) 355

Pinto Coelho Silvestre, Marialice . . . . . (1) 5

Piva, Andrea . . . . . . . . . . . . . (2) 89

Plou, Francisco J.. . . . . . . . . . . . . . . . . (4) 337

Porto, André L. M. . . . . . . . . . . . (4) 355

Rakin, Marica . . . . . . . . . . . . . (2) 109

Ramírez, José A. . . . . . . . . . . . $(1,2) \quad 1,75$

Ratola, Nuno . . . . . . . . . . . . . (2) 125

Reddy, Gopal . . . . . . . . . . . . . (3) 147

Reymond, Jean-Louis . . . . . . . . . . (4) 265

Ribeiro Dias, Disney . . . . . . . . . . (1) 5

Ribeiro Vianna, Fabiana . . . . . . . . . (1) 5

Rodrigues, J. Augusto R. . . . . . . . . (4) 295

Rodríguez-Monroy, Mario . . . . . . . . (3) 153

Ruzzi, Maurizio . . . . . . . . . . . (3) 193

Sak-Bosnar, Milan . . . . . . . . . . . (3) 197,207

Sánchez-Montero, José María . . . . . . (4) 343

Sant'Anna, Ernani S. . . . . . . . . . . . (1) 41

Schaper Bizzotto, Carolina. . . . . . . . (1) 5

Șekeroğlu, Gülten . . . . . . . . . . . . (1) 27

Serviene, Elena

Seyis, Isil . . . . . . . . . . . . . . . . . . (2) 121

Shaban, Gehan M. . . . . . . . . . . . (2) 83

Silva, Carla J. S. M.. . . . . . . . . . . . . . (1) 51
Sinisterra, José Vincente. . . . . . . . . (4) 343

Skowronek, Marcin. . . . . . . . . . . (3) 141

Sobočanec, Sandra . . . . . . . . . . . . . (3) 189

Sone, Teruo . . . . . . . . . . . . . . . . . (3) 169

Sousa, Fernanda . . . . . . . . . . . . (1) 51

Stanković, Snežana . . . . . . . . . . . (3) 183

Stanzer, Damir. . . . . . . . . . . . . (2) 115

Stehlik-Tomas, Vesna . . . . . . . . . . (2) 115

Steiner, Walter . . . . . . . . . . . . . (4) 219,223

Stocchi, Vilberto . . . . . . . . . . . . (1) 57

Su, Xinguo . . . . . . . . . . . . . . . . . (2) 131

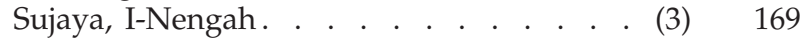

Suzzi, Giovanna . . . . . . . . . . . . (2) 89

Šverko, Višnja . . . . . . . . . . . . . . (3) 189

Tarakçı, Zekai . . . . . . . . . . . . . (1) 47

Tedesco, Idolo . . . . . . . . . . . . . . . . . . . (1) 37

Teixeira, José A. . . . . . . . . . . . . . . . (4) 327

Téllez-Luis, Simón J. . . . . . . . . . . . $(1,2) \quad 1,75$

Torre, Paolo . . . . . . . . . . . . . . . . (3) 193

Torres, Sebastián . . . . . . . . . . . . (4) 271

Trejo-Espino, José Luis . . . . . . . . . (3) 153

Trejo-Tapia, Gabriela . . . . . . . . . . (3) 153

Tunçtürk, Yusuf . . . . . . . . . . . . (1) 47

Vadnjal, Robert . . . . . . . . . . . . (3) 175

Vahčić, Nada . . . . . . . . . . . . . (2) 115

Vallorani, Luciana . . . . . . . . . . . (1) 57

Vázquez, Manuel . . . . . . . . . . . . $(1,2) \quad 1,75$

Villarreal, Ma. Luisa . . . . . . . . . . (3) 153

Vukasinovic, Maja . . . . . . . . . . . (2) 109

Vuyani Mabinya, Leonard . . . . . . . . (1) 23

Waché, Yves. . . . . . . . . . . . . . (4) 327

Wang, Yuehua . . . . . . . . . . . . . (2) 135

Weis, Roland . . . . . . . . . . . . . (4) 237

Wondra, Mojmir . . . . . . . . . . . . (1) 11

Zechner-Krpan, Vesna. . . . . . . . . . (3) 213

Živković, Jelena . . . . . . . . . . . . . . (3) 183 


\section{SUBJECT INDEX}

\section{A}

$\alpha$-galactosidase

from Erwinia chrysanthemi, 23

degradation of melibiose and raffinose, 23

kinetic properties of, 23

$\alpha, \beta$-unsaturated carbonyl compounds

biocatalytic asymmetric reduction of, 295

acetophenones

biocatalytic asymmetric reduction of, 295

acidophiles

the biocatalytic potential of, 223

biomass and enzyme production, 223

cloning and expression of their genes in mesophilic hosts, 223

adrenalin test

for screening of enzyme activities, 265

aldehydes algae

biocatalytic asymmetric reduction of, 295

as food supplements, 57

marine algae, Wakame and Kombu, 57

protein quality, 57

vitamin and mineral composition, 57

alkaline phosphatase

inactivation by temperature and $\mathrm{CO}_{2}$ treatment, 27

effect of raw milk on its stability during $\mathrm{CO}_{2}$

treatment, 27

alkaliphiles

the biocatalytic potential of, 223

biomass and enzyme production, 223

cloning and expression of their genes in

mesophilic hosts, 223

alkene monooxygenase

from Trichosporum cutaneum, 355

amperometric surfactant sensors

in biotechnology, 197

amylolytic strain

Lactobacillus amylophilus for lactic acid production on wheat bran, 147

Amylomyces rouxii

in fermented potato pulp ragi tapé, 169

anoxia treatment

of litchi fruit to extend its shelf life, 131

anthocyanins

in Cabernet Sauvignon and Merlot, 11

non-anthocyanic flavonoids in red wine, 37

impact on the young red wine colour, 183

anticancer agents

biocatalytic synthesis of chiral pharmaceutical

intermediates, 305

antihypertensive agents

biocatalytic synthesis of chiral pharmaceutical intermediates, 305

anti-infective drugs

biocatalytic synthesis of chiral pharmaceutical

intermediates, 305 antioxidant capacity

of clinoptilolite mixture with Urtica dioica L. extract, 189

antioxidants

polyphenolic, from wine, 11, 37, 89

polyphenolic, from carobs, 105

antiradical activity

of polyphenols from carobs, 105

antiviral agents

biocatalytic synthesis of chiral pharmaceutical intermediates, 305

aroma compounds

during fermentation of grape must, 11, 37, 89

production of ionones from carotenoids by

enzymatic, thermal or microbial degradation, 327

generation of green notes by

lipoxygenase/hydroperoxide lyase pathway, 327

pathway of production through $\beta$-oxidation

( $\gamma$-decalactone), 327

Aspergillus niger

production of citric acid, 19

undersized semolina as growth substrate, 19

inulinase production using simplex and classical method, 141

Baeyer-Villiger monooxygenase activity in, 355

Aspergillus oryzae avian

Baeyer-Villiger monooxygenase activity in, 355

monoclonal antibodies recognizing its IgY

epitopes, 175

\section{B}

$\beta$-1,3-glucanase

production by free and immobilised Trichoderma harzianum, 83

effect of benomyl on its production, 83

$\beta$-oxidation ( $\gamma$-decalactone)

pathway of aroma compounds production, 327

Baeyer-Villiger monooxygenase activity

monitoring by biocatalytic method and

multibioreaction approach, 355

in Geotrichum candidum, 355

in Aspergillus oryzae, 355

in Curvularia lunata, 355

in Aspergillus niger, 355

in Trichoderma sp., 355

in Cunninghamella echinulata, 355

beetroot juice fermentation

by lactic acid bacteria, 109

influence of brewer's yeast autolysate on, 109

as functional food additive, 109

benomyl

effect on chitinase and $\beta-1,3$-glucanase production by Trichoderma harzianum, 83 
bentonite

impact on coloured matters of young red wines, 183

binding properties

water holding capacity in ham pâté, 5

raw batter stability in ham pâté, 5

influence of bovine blood on, 5

biocatalysis

synthesis of dextran by dextransucrases, 337

with lipases from Candida rugosa, 343

resolution of racemic mixtures, 343

organic synthesis in slightly hydrated organic

solvents, 343

Baeyer-Villiger monooxygenase activity, 355

asymmetric reduction of acetophenones and

$\alpha, \beta$-unsaturated carbonyl compounds, 295

production of aroma compounds from lipids, 327

in homogeneous solvent systems, 271

with hydroxynitrile lyases, 287

of chiral pharmaceutical intermediates, 305

with extremophiles and extremozymes, 223

in non-conventional heterogeneous media, 279

strategies for improving enzymes for, 251

with enzymes from higher eukaryotes, 237

biocatalyst engineering

protein engineering, 251

directed evolution, 251

medium engineering, 251

substrate engineering, 251

biocatalyst discovery and optimisation, 251

biocontrol

effect of benomyl on enzyme production by

Trichoderma harzianum, 83

biogenic amines

in sucuk, 63

biosensors

amperometric surfactant sensors that use living cells, 197

biotransformations

synthesis of dextran by dextransucrases, 337

with lipases from Candida rugosa, 343

resolution of racemic mixtures, 343

Baeyer-Villiger monooxygenase activity, 355

asymmetric reduction of acetophenones and

$\alpha, \beta$-unsaturated carbonyl compounds, 295

aroma compounds production from lipids, 327

with hydroxynitrile lyases, 287

biological catalysts within a multiphase medium, 327

of synthetic chemicals with high chemo-, regio-

and enantioselectivities, 305

with extremophiles and extremozymes, 223

in non-aqueous media, 271, 279

strategies for improving enzymes for, 251

enzymes from higher eukaryotes for industrial

biocatalysis, 237

bovine blood

as a fat replacer in ham pâté, 5

influence on salt-soluble proteins in ham pâté, 5

broccoli effect of hot water immersion on its storage

quality, 135

\section{C}

Ca-alginate

encapsulation of Trichoderma harzianum, 83

encapsulation of dextransucrases, 337

Cabernet Sauvignon

production of, 11

phenolic compounds in, 11

anthocyanins in, 11

tone and colour intensity, 11

Candida rugosa

optimal productivity of extracellular lipases from, 343 carobs

as a source of polyphenolic antioxidants, 105

carotenoids

production of ionones from, by enzymatic, 327

thermal or microbial degradation, 327

carrot root

guaiacol peroxidases in, 33

casein

in growth media for transglutaminase production

by Streptoverticillium ladakanum, 75

cheese

fresh and ripened herby cheese (Otlu peynir), 47

chemoselectivities

in biotransformations of synthetic chemicals, 305

chiral building blocks

for fine chemicals and pharmaceutical industries, 295

chiral pharmaceutical intermediates

biocatalytic synthesis of, 305

for antiviral agents, 305

for anti-infective drugs, 305

for anticancer agents, 305

for antihypertensive agents, 305

receptor agonists/antagonists, 305

enantioselective enzymatic de-protection, 305

chitinase

production by free and immobilised Trichoderma

harzianum, 83

effect of benomyl on its production, 83

citric acid

production by Aspergillus niger, 19

undersized semolina as substrate for its

production, 19

clinoptilolite

natural, micronised, 189

in mixture with Urtica dioica L. extract, 189

its antioxidant capacity, 189

colour intensity

in Cabernet Sauvignon and Merlot, 11

influence of wine yeasts on, 37

bentonite and gelatine impact on, in young red wine, 183

conductometric surfactant sensors

in biotechnology, 197

copper

yeast enriched with, 115 
copper-calcein assay

for detecting amino acids, 265

crosslinking

of enzyme esperase, albumin and casein with glutaraldehyde, 51

Cunninghamella echinulata

Baeyer-Villiger monooxygenase activity in, 355

Curvularia lunata

Baeyer-Villiger monooxygenase activity in, 355

cyanohydrin synthesis

catalysed by hydroxynitrile lyases, 287

enantioselective reaction, 287

\section{D}

Daucus carota

guaiacol peroxidases in, 33

dextran

modulating the synthesis of, using native and encapsulated dextransucrases, 337

dextransucrases

modulating the synthesis of dextran with, 337

encapsulation in alginate, 337

direct fermentation

of wheat bran for lactic acid production by

Lactobacillus amylophilus, 147

directed evolution

in biocatalyst engineering, 251

dot immunobinding assay

for detection of specific IgY antibodies, 175

dry-fermented sausages

quality characteristics of sucuk, 63

biogenic amines in, 63

microbial composition, 63

dsRNAs

responsibility for killer activity of wine yeasts, 159

\section{E}

electric surfactant sensors

in biotechnology, 207

electrochemical surfactant sensors

potentiometric, 197

ISFET sensors, 197

for flow injection analysis (FIA), 197

amperometric, 197

conductometric, 197

impedimetric, 197

enantioselective enzymatic de-protection

in biocatalytic synthesis of chiral pharmaceutical

intermediates, 305

enantioselectivities

in biotransformations of synthetic chemicals, 305

encapsulation

of Trichoderma harzianum in Ca-alginate, 83

of dextransucrases in Ca-alginate, 337

Erwinia chrysanthemi

kinetic properties of $\alpha$-galactosidase from, 23

localization of total proteins in, 23

Escherichia coli

production of vanillin from ferulic acid, 193 non-specific oxidase activity, 193

esperase

crosslinking with glutaraldehyde, 51

extremophiles

the biocatalytic potential of, 223

biomass and enzyme production, 223

cloning and expression of their genes in

mesophilic hosts, 223

thermophiles, 223

psychrophiles, 223

alkaliphiles, 223

acidophiles, 223

halophiles, 223

piezophiles, 223

metallophiles, 223

xerophiles, 223

extremozymes

the biocatalytic potential of, 223

cloning and expression of their genes in

mesophilic hosts, 223

from thermophiles, 223

from psychrophiles, 223

from alkaliphiles, 223

from acidophiles, 223

from halophiles, 223

from piezophiles, 223

from metallophiles, 223

from xerophiles, 223

\section{F}

fat replacer

bovine blood in ham pâté, 5

influence on salt-soluble proteins in ham pâté, 5

influence on binding properties in ham pâté, 5

fermentation

of grape must, 11, 37, 89, 165

of cultivars Cabernet Sauvignon and Merlot, 11

production of citric acid by Aspergillus niger, 19

of tuna meat with Lactobacillus casei subsp. casei, 41

of beetroot juice, 109

of standard molasses substrate and enrichment of yeast with microelements, 115

of sucuk sausages, 63

of wheat bran for lactic acid production by

Lactobacillus amylophilus, 147

of potato pulp with Indonesian ragi tapé, 169

ferulic acid

for production of vanillin by Escherichia coli, 193 fining

of young red wine by bentonite and gelatine, 183

flow injection analysis (FIA)

surfactant sensors for, 197

fluorogenic assay

for screening of enzyme activities, 265

food supplements

marine algae Wakame and Kombu, 57

functional food additive

beetroot juice fermented by lactic acid bacteria, 109 
fungicides

benomyl effect on enzyme production by

Trichoderma harzianum, 83

\section{G}

gelatine

impact on coloured matters of young red wines, 183

Geotrichum candidum

Baeyer-Villiger monooxygenase activity in, 355

glucosyltransferases

modulating the synthesis of dextran with, 337

encapsulation in alginate, 337

glutaraldehyde

for crosslinking of enzyme esperase, albumin and casein, 51

glycerol

in growth media for transglutaminase production

by Streptoverticillium ladakanum, 75

green notes

biotechnological production by

lipoxygenase/hydroperoxide lyase pathway, 327

expression of plant hydroperoxide lyase-encoding genes in microorganisms, 327

guaiacol peroxidases

from carrot root, 33

stress influence on its expression, 33

\section{$\mathbf{H}$}

halophiles

the biocatalytic potential of, 223

biomass and enzyme production, 223

cloning and expression of their genes in mesophilic hosts, 223

ham pâté

bovine blood as fat replacer in, 5

salt-soluble proteins in, 5

binding properties in, 5

Hanseniaspora uvarum

biodiversity of natural population in wine, 165

herby cheese

fresh and ripened, 47

chemical and biochemical properties of, 47

heterogeneous solvent systems

non-aqueous biocatalysis in, 279

reverse micelles in, 279

supercritical fluids, 279

ionic liquids, 279

higher eukaryotes enzymes from, for industrial biocatalysis, 237

homogeneous aqueous-organic mixtures

enzyme stability and activity in, 271

solubility of enzymes in, 271

enzyme-solvent interactions, 271

hot water immersion

of fresh broccoli heads, 135

hydroxynitrile lyases

biological sources, 287 enantioselectivity of, 287

in cyanohydrin synthesis, 287

genetically modified, 287

$\operatorname{Ig} Y$

epitopes shared by Phasianidae birds, 175

recognition by monoclonal antibodies, 175

immobilisation

Ca-alginate encapsulation of Trichoderma harzianum, 83

Ca-alginate encapsulation of dextransucrases, 337

impedimetric surfactant sensors

in biotechnology, 197

inactivation

of alkaline phosphatase by temperature and $\mathrm{CO}_{2}$ treatment, 27

inactivation

of maltose transport and metabolism in

Saccharomyces cerevisiae, 213

induction

of maltose transport and metabolism in

Saccharomyces cerevisiae, 213

inulinase

production by Aspergillus niger using simplex and

classical method, 141

ionic liquids

in heterogeneous solvent systems, 279

ionones

production from carotenoids by enzymatic, thermal or microbial degradation, 327

generation by lipoxygenase action, 327

generation by the action of xanthine oxidase, 327

ISFET surfactant sensors

in biotechnology, 197

isoenzymes

produced by Candida rugosa, 343

used as biocatalysts, 343

K

ketones

biocatalytic asymmetric reduction of, 295

killer activity

of yeasts in fruit and berry wine, 159

dsRNAs responsibility for, 159

Kombu

protein quality, 57

vitamin and mineral composition, 57

L

$\mathrm{L}(+)$ lactic acid production by Lactobacillus amylophilus, 147

production in wheat bran, 147

direct fermentation, 147

lactase

production by Trichoderma sp., 121 
$\mathrm{pH}$ and temperature stability, 121

lactic acid bacteria

in fermentation of dark tuna meat, 41

in sucuk, Turkish dry-fermented sausages, 63

influence on beetroot juice fermentation, 109

production of $\mathrm{L}(+)$ lactic acid, 147

Lactobacillus acidophilus

in beetroot juice fermentation, 109

Lactobacillus amylophilus GV6

production of $\mathrm{L}(+)$ lactic acid, 147

semi-solid state fermentation using wheat bran,

147

Lactobacillus casei subsp. casei

in fermentation of dark tuna meat, 41

Lactobacillus plantarum

in beetroot juice fermentation, 109

landfill leachate

organic composition of, 99

nanofiltration for the purification of, 99

Leuconostoc mesenteroides

dextransucrases from, 337

lipase

from Candida rugosa, 343

resolution of racemic acids, esters and amines by, 343

purification, 343

lipid degradation bioreactors lipids

mass transfer within multiphase systems, 327

for aroma compounds production, 327

biological catalysts within a multiphase medium, 327 Litchi chinensis

anoxia treatment of, for shelf life extension, 131

$\mathbf{M}$

maltose

transport and metabolism in Saccharomyces

cerevisiae, 213

regulation mechanisms in Saccharomyces cerevisiae, 213

manganese

yeast enriched with, 115

medium engineering

for improving enzymes for efficient biocatalysis, 251 melibiose

as substrate for $\alpha$-galactosidase from Erwinia

chrysanthemi, 23

membrane technology

in leachate treatment procedures, 99

Merlot wine

production of, 11

phenolic compounds in, 11

anthocyanins in, 11

tone and colour intensity, 11

metabolism

of maltose and its effect on gene expression in

Saccharomyces cerevisiae, 213

metal uptake

with Saccharomyces cerevisiae, 115

metalophiles

the biocatalytic potential of, 223 biomass and enzyme production, 223

cloning and expression of their genes in mesophilic hosts, 223

method validation

for analysis and quantification trans-resveratol, using HPLC, 125

milk

alkaline phosphatase stability in, 27

monoclonal antibodies

useful in diagnostic and avian and mammalian

infections, 175

production, characterization and use, 175

recognized IgY epitopes shared by chicken, turkey,

pheasant, peafowl and sparrow, 175

multibioreaction methodology

in monitoring of Baeyer-Villiger monooxygenase activity, 355

\section{$\mathbf{N}$}

nanofiltration

of landfill leachate containing pharmaceutical

intermediates, 99

non-conventional heterogeneous media

non-aqueous biocatalysis in, 279

reverse micelles in, 279

supercritical fluids, 279

ionic liquids, 279

non-electrochemical surfactant sensors

optochemical, 207

electric, 207

piezoelectric, 207

\section{O}

optochemical surfactant sensors

in biotechnology, 207

organic matter

in landfill leachate purified by nanofiltration, 99

organic solvents

in non-aqueous biocatalysis in homogenous

solvent systems, 271

enzyme stability and activity in, 271

solubility of enzymes in, 271

enzyme-solvent interactions, 271

in non-aqueous biocatalysis in non-conventional heterogeneous media, 279

Otlu peynir

fresh and ripened herby cheese, 47

chemical and biochemical properties of, 47

oxidoreductase

from yeast, 295

reduction of acetophenones and $\alpha, \beta$-unsaturated carbonyl compounds, 295

oxynitrilase

biological sources, 287

enantioselectivity of, 287

in cyanohydrin synthesis, 287

genetically modified, 287 
PCR-denaturing gradient gel electrophoresis for identification of microflora from fermented potato pulp, 169

pesticide residues

influence on $S$. cerevisiae fermentation of grape must, 89

pharmaceutical chemicals

in landfill leachate purified by nanofiltration, 99

Phasianidae birds

monoclonal antibodies recognizing its IgY epitopes, 175

phenols

in Cabernet Sauvignon and Merlot, 11

influence of yeasts on polyphenol composition of wine, 37,89

piezoelectric surfactant sensors

in biotechnology, 207

piezophiles

the biocatalytic potential of, 223

biomass and enzyme production, 223

cloning and expression of their genes in mesophilic hosts, 223

plant cell morphology

of Solanum chrysotrichum, 153

polyphenols

composition in wine, $11,37,89$

from carobs, 105

as antioxidants, 105

its antiradical and reducing properties, 105

potato pulp

fermented with Indonesian starter ragi tapé, 169

potentiometric surfactant sensors

in biotechnology, 197

prebiotics

synthesis of metyl polyglucosides with

dextransucrases, 337

protein engineering

for improving enzymes for efficient biocatalysis, 251

rational design, 251

proteins

salt-soluble proteins in ham pâté, 5

chemical modifications on, using glutaraldehyde, 51

quality of marine algae Wakame and Kombu, 57

psychrophiles

the biocatalytic potential of, 223

biomass and enzyme production, 223

cloning and expression of their genes in

mesophilic hosts, 223

\section{Q}

quinoxyfen

influence on $S$. cerevisiae fermentation of grape must, 89

\section{$\mathbf{R}$}

raffinose

as substrate for $\alpha$-galactosidase from Erwinia chrysanthemi, 23

ragi tapé

microflora and selected metabolites of, 169

rapid fluorescence methodology

in monitoring of Baeyer-Villiger monooxygenase activity, 355

rational design

for improving enzymes for efficient biocatalysis, 251

receptor agonists/antagonists

in biocatalytic synthesis of chiral pharmaceutical intermediates, 305

reducing power

of polyphenols from carobs, 105

regioselectivities

in biotransformations of synthetic chemicals, 305

regulation mechanisms

of maltose transport and metabolism in

Saccharomyces cerevisiae, 213

induction, 213

glucose repression, 213

glucose inactivation, 213

repression

of maltose transport and metabolism in

Saccharomyces cerevisiae, 213

resolution of racemics

by Candida rugosa lipase in slightly hydrated

organic solvents, 343

response surface method

for optimisation of lactic acid production by

Lactobacillus amylophilus, 147

resting cells

of Escherichia coli, in vanillin production from ferulic acid, 193

reverse micelles

in heterogeneous solvent systems, 279

rheological properties

of growth media of Solanum chrysotrichum, 153

Rhizopus-like fungi

in fermented potato pulp ragi tapé, 169

\section{S}

Saccharomyces cerevisiae

for winemaking of must from grapes, 37, 89

influence of quinoxyfen residues on grape

fermentation by, 89

enrichment with zinc, copper and manganese, 115

killer yeasts in fruit and berry wine, 159

dsRNA extraction, 159

killer toxins production, 159

biodiversity of natural population in wine, 165

regulation of maltose transport and metabolism in, 213

salt-soluble proteins

content in ham pâté, 5

influence of bovine blood on, 5 


\section{SDS-PAGE}

of the samples of casein, esperase and albumin aggregates, 51

and immunoblot assay in recognition of specific IgY antibodies, 175

semolina (undersized)

as growth substrate for Aspergillus niger, 19

for production of citric acid, 19

simplex optimisation

of inulinase production by Aspergillus niger, 141

size-exclusion chromatography

of the samples of casein, esperase and albumin aggregates, 51

Solanum chrysotrichum

morphological properties, 153

rheological properties of its growth media, 153

comparison of growth in stirred tank bioreactor and in shake flask, 153

solid phase microextraction - capillary gas

chromatography (SPME-GC)

analysis of volatile compounds in grape must, 89

sorghum straw hydrolysates

media for growth of Streptoverticillium ladakanum, 1

for production of transglutaminase, 1

spectrophotometric enzyme assays

for high-throughput screening, 265

storage treatment

of litchi fruit with anoxia, to extend its shelf

life, 131

of fresh broccoli heads with hot water immersion, 135

Streptoverticillium ladakanum

production of transglutaminase, 1

growth on hydrolysates of sorghum straw, 1

production of transglutaminase by, 75

optimisation of growth media, 75

substrate engineering sucuk

for improving enzymes for efficient biocatalysis, 251

quality characteristics of, 63

biogenic amines in, 63

microbial composition, 63

supercritical fluids

in heterogeneous solvent systems, 279

surfactant sensors

in biotechnology, 197, 207

electrochemical, 197

non-electrochemical, 207

surfactants

application in modern biotechnology, 197, 207

synthetic chemicals

biotransformations of, with high chemo-, regioand enantioselectivities, 305

\section{$\mathbf{T}$}

thermal stability

of alkaline phosphatase in buffer and milk, 27

thermophiles

the biocatalytic potential of, 223

biomass and enzyme production, 223 cloning and expression of their genes in

mesophilic hosts, 223

thin-layer chromatography (TLC) based screening of enzyme activities, 265

transglutaminase

production by Streptoverticillium ladakanum

NRRL-3191, 1, 75

transport

of maltose in Saccharomyces cerevisiae, 213

trans-resveratol

in wines, 125

analysis and quantification of, by HPLC, 125

Trichoderma harzianum

Ca-alginate encapsulation of, 83

effect of benomyl on chitinase and $\beta$-1,3-glucanase production, 83

Trichoderma sp.

lactase production by, 121

Baeyer-Villiger monooxygenase activity in, 355

Trichosporum cutaneum

alkene monooxygenase in, 355

tuna meat

fermented with Lactobacillus casei subsp. casei, 41

$\mathbf{U}$

urea-PAGE

of fresh and ripened herby cheese, 47

Urtica dioica $\mathrm{L}$.

extract with clinoptilolite as possible antioxidants, 189

\section{V}

vanilic acid

production by Escherichia coli, from ferulic acid, 193

vanillin

production by Escherichia coli from ferulic acid, 193 oxidation to vanilic acid, 193

\section{W}

Wakame

protein quality, 57

vitamin and mineral composition, 57

wheat bran

as substrate for lactic acid production by

Lactobacillus amylophilus, 147

wine

fermentation of cultivars Cabernet Sauvignon and Merlot, 11

phenolic compounds in, 11, 37, 89

anthocyanins in, 11

tone and colour intensity, 11, 37

wine yeasts, 37, 89

trans-resveratol analysis and quantification in, 125

occurrence of killer yeasts in fruit and berry wine, 159 
biodiversity of natural yeast population in wine, 165

bentonite and gelatine impact on young red wine colour, 183

\section{$\mathbf{X}$}

xerophiles

the biocatalytic potential of, 223

biomass and enzyme production, 223

cloning and expression of their genes in mesophilic hosts, 223

\section{$\mathbf{Y}$}

yeasts

for winemaking of must from grapes, 37, 89 autolysates in beetroot juice fermentation by lactic acid bacteria, 109

enrichment with zinc, copper and manganese, 115

killer yeasts in fruit and berry wine, 159

dsRNA extraction, 159

killer toxins production, 159

biodiversity of natural population in wine, 165

regulation of maltose transport and metabolism in, 213

used as biocatalysts for redox reactions of various functional groups, 295

zinc

yeast enriched with, 115 